\title{
Difference making, explanatory relevance, and mechanistic models*
}

\author{
Dingmar van EcK and Raoul Gervais
}

Received: 18/12/2014

Final Version: 06/03/2015

BIBLID 0495-4548(2016)31:1p.125-134

DOI: $10.1387 /$ theoria. 13282

ABSTRACT: In this paper we consider mechanistic explanations for biological malfunctions. Drawing on Lipton's (1993) work on difference making, we offer three reasons why one should distinguish i) mechanistic features that only make a difference to the malfunction one aims to explain, from ii) features that make a difference to both the malfunction and normal functioning. Recognition of the distinction is important for a) repair purposes, b) mechanism discovery, and c) understanding. This analysis extends current mechanistic thinking, which fails to appreciate the distinction. We illustrate our contribution with a case on sleeping disorders as arising from disruptions of circadian rhythms.

Keywords: mechanistic explanation; difference making; explanatory relevance; malfunction explanation.

RESUMEN: En este artículo tomamos en consideración las explicaciones mecanicistas de las disfunciones biológicas. A partir del trabajo de Lipton (1993) sobre diferenciación (difference making), damos tres razones por las que se debería distinguir entre: i) aspectos mecanicistas que sólo suponen una diferencia para la disfunción que se pretende explicar, y ii) aspectos que suponen una diferencia tanto para la disfunción como para el funcionamiento normal. Reconocer la distinción tiene importancia para: a) fines de reparación, b) el descubrimiento de mecanismos, y c) la comprensión. Este análisis extiende el pensamiento mecanicista actual, que no llega a percibir la distinción. Ilustramos nuestra contribución con un caso de desorden del sueño que surge de disrupciones de los ritmos circadianos.

Palabras clave: explicación mecanicista, diferenciación, relevancia explicativa, explicación de las disfunciones.

\section{Introduction}

In the literature on explanation in the life sciences, it is now uncontested that mechanisms play a central role in explaining capacities such as digestion, pattern recognition, or the maintenance of circadian rhythms. The idea is that to explain such capacities, one provides a model, or more generally a description/representation, of the mechanism responsible for that capacity. But what features of the mechanism should we include in our model? The obvious answer is that we should include only relevant details. But then what constitutes relevance? Although the details vary from author to author, roughly, the idea is that those features that make a difference to the explanandum are relevant (Craver 2007; Levy and Bechtel 2013; cf. Waters 2007). Yet, what does 'making a difference' precisely mean? Opinions diverge. Craver $(2007,152)$ hitches his mutual manipulability account of consti-

* The authors thank two anonymous referees, and the members of the Tilburg Center for Logic, Ethics, and Philosophy of Science, for their comments on earlier versions of this paper. 
tutive relevance to Woodward's (2003) account of (causal) explanation. On this construal, mutual manipulability relations comprise both constitutive relevance relations with respect to the occurrence of explananda phenomena, and relations concerning the precise manner in which explananda phenomena occur (van Eck 2015a). Levy and Bechtel (2013), in contrast, tie their notion of difference making to Strevens' $(2004,2008)$ system, at least in the context of explaining how the behaviour of mechanisms results from the organization of mechanisms. On Strevens' account, and hence Levy and Bechtel's system as well, explanatory models should refer only to those features that make a difference with respect to the occurrence of specific explananda phenomena. ${ }^{1}$

So we have two ways to understand explanatory relevance and difference making: on the one hand "primary causal factors" that make a difference to occurrence and on the other "higher order causal factors" that only affect how it occurs (cf. Weisberg 2007, 651). We believe, however, that this story is incomplete. We will argue that in the context of mechanistic malfunction explanation, the distinction between primary and higher order factors that make a difference to the explanandum is too simple. Drawing on Peter Lipton's (1993) framework, we argue that one can distinguish three features of a mechanism: i) those that make a difference to the malfunction and not to the normal functioning of a mechanism, ii) those that make a difference to both the malfunction and to the normal functioning, and iii) those that make a difference to normal function and not to malfunction. ${ }^{2}$ While we are obviously interested in the first category, we argue that factors of the second category are also highly relevant: explicit recognition of the distinction between (first category) contrastive and (second category) common difference making factors aids in mechanism discovery, serves repair or treatment purposes, and facilitates understanding. We illustrate our claims with a case study: the explanation of sleeping disorders as resulting from disruptions of human circadian rhythms. ${ }^{3}$

1 To be sure, in Strevens' $(2004,2008)$ system, explananda phenomena may comprise many things, such as events, properties, and regularities. What is crucial in Strevens' system is that explanatory models should only refer to those factors that are crucial for the explanatory target to obtain, i.e., to occur, whether it be a specific event, a regularity, a property, or something else. Strevens $(2004,158)$ himself puts it thus: "the explanatorily relevant parts of any causal network are the elements that made a difference to whether or not the explanandum occurred. It is important to note the whether or not. To be explanatorily relevant, a causal factor must not merely make a difference to how the explanandum occurred; it must make a difference large enough to bear on whether or not it occurred at all."

2 Of course, features that make a difference to a malfunction may start making a difference to normal function when they are sufficiently intervened upon, e.g., fixing a rupture in a car's oil reservoir which prevents it to continue leaking oil. Also, importantly, features that make a difference to both normal function and malfunction are of the higher order variety in the sense elaborated by Weisberg: they concern normal vs. impaired performance of capacities that are present, say, normal vision vs. impaired vision, not the manifestation vs. absence of capacities, say, normal vision vs. blindness. There are no features of course that at the same time make a difference to the manifestation and absence of a given capacity (see note 6).

3 Some might object at this point that the proposed distinction between contrastive and common difference makers is implicitly present in accounts of mechanistic explanation and that explicit recognition is unnecessary. The distinction is not to be had for free though. On the mechanistic view, items/constituents either fulfill a role function and thereby contribute to overall mechanism function, or items/constituents fail to fulfill a role function and thereby contribute to overall malfunction. This conceptualiza- 
Let us conclude with an overview. We discuss Lipton's framework in section two, after which we present current mechanistic thinking on explanatory relevance in section three. We then elaborate Lipton's framework in term of a detailed case study on mechanistic explanations of sleeping disorders and disrupted human circadian rhythms and wrap up our discussion in section four.

\section{Making a difference, but to what?}

Many explanatory questions about malfunctions are contrastive - why does something malfunction, rather than function normally? If we combine this with what we said about capacities in the introduction, we can say that the general format of such questions is as follows: "Why does system $S$ fail to perform capacity C, rather than perform it?" Examples include: "Why is my eyesight impaired?", "Why does John's digestive system not work properly?", or "Why do elderly people often have hearing problems?" These examples illustrate two further points: often, the foil is left implicit, and second, we might desire explanations for a particular malfunction, or for a type of malfunction, as the last example indicates. Moreover, fact and foil may concern occurrences vs. non-occurrences - e.g., why does this electric screwdriver fail to drive screws at all- or differences in performance, as in the above examples where capacities are present yet impaired.

So how do we answer questions like these? In the case of the capacities studied by the life sciences, adequately answering them requires knowledge about the underlying mechanisms. However, not every aspect of a mechanism (entities, activities/operations, and organizational features) will be relevant. As said in the introduction, the most common way to understand relevance here is that the parts of the mechanism that are cited by the explanation should make a difference to the explanandum - in our case, either the absence or suboptimal performance of a capacity. But again, some parts will make a difference, not only to the malfunction, but also to the normal functioning of the system. ${ }^{4}$ This point was noted by Peter Lipton, who gave a particularly clear example:

Suppose that my car is belching thick, black smoke. Wishing to correct the situation, I naturally ask why it is happening. Now imagine that God (or perhaps an evil genius) presents me with a full Deductive-Nomological explanation of the smoke. This may not be much help. The problem is that many of the causes of the smoke are also causes of the car's normal operation. Were I to eliminate one of these, I might only succeed in making the engine inoperable. By contrast, an

tion blurs the fact that some items/constituents fulfilling a role contribute to both normal function and malfunction. To the best of our knowledge, mechanistic theories of function do not distinguish/recognize that the same components may contribute to malfunction as well as to normal function (cf. Craver 2001, 2013; Illari \& Williamson 2010). Such recognition is relevant for several explanatory tasks as we argue in this paper. We thank an anonymous referee for urging us to elaborate on this issue.

4 Hence, not all explanatory work is or can be done by current mechanistic accounts of 'normal' function since these accounts do not invoke conceptual machinery to recognize/distinguish that the same components may contribute to malfunction as well as to normal function (cf. Craver 2001, 2013; Illari \& Williamson 2010). Not incidentally, mechanistic accounts of constitutive relevance also fail to (explicitly) recognize the distinction between contrastive and common factors (e.g., Craver 2007; Harbecke 2010; Couch 2011; Baumgartner \& Gebharter 2015). 
explanation of why the car is smoking rather than running normally is far more likely to meet my diagnostic needs (1993, 53, italics removed).

Now, in this quote, Lipton is explicitly comparing contrastive causal explanations with deductive-nomological explanations, but the example he gives can also be used to clarify what is conceptually at stake here. There are basically four types of factors: those that make a difference to the smoke, but not to the normal operation (e.g., an oil leakage), those that make a difference both to the smoke and the normal operation (e.g., the carburettor producing sparks), those that make a difference to normal operation and not to the smoke (e.g., the wheels turning), and those that make a difference to neither (e.g., the windscreen wipers). ${ }^{5}$ It is obvious that we are particularly interested in the first category, since, if our goal is to explain the malfunction we want to know the factor(s) that marks the contrast between malfunction and normal function. Similarly, if our goal is fix the malfunction, we do not want to interfere with common factors of the second category, e.g., the engine's normal operation.

Note that in Lipton's example fact and foil are rather awkwardly defined. Lipton (1993) asks why his car is belching black smoke rather than running normally. A more obvious foil of the presence of thick, black smoke would be something like 'the normal amount of smoke'. Likewise, if 'running normally' is the foil, a more obvious fact would be 'not running at all' or 'not running smoothly'. In the case of capacities that are impaired - like the car not running smoothly - we take it that common factors of the second category are also very relevant. ${ }^{6}$

We consider three reasons for this claim. First, as already suggested above, distinguishing contrastive difference makers from factors common to both malfunction and normal functioning is relevant for repair (or therapeutic intervention) purposes, since you only want to tinker with factors of the first category. Lipton is right that in correcting the situation of the smoke, we do not want to intervene upon those common factors. Similarly, repairs (or therapeutic interventions) can only be made, and similarly, explanations provided, when a malfunctioning mechanism is individuated properly, that is, when both contrastive and common factors have been identified and the malfunction (contrastive factor) has been localized in a mechanism (cf. Thagard 2003; Moghaddam-Taaheri 2011). Finally, the contrastive-common distinction procures understanding in the sense of offering means for answering what-if-things-had-been-different-questions (Woodward 2003).

For instance, oil leaking (onto the hot exhaust) might be the contrastive factor explaining the black smoke. One then may want to intervene on the oil leakage but not on the ex-

5 Other factors being equal of course; if we mess with other factors, the cited factors may or may not make a difference depending on the nature of the intervention.

6 Note that we restrict our analysis to capacities that are present, yet impaired. If fact and foil are defined exclusively in terms of occurrence vs. non-occurrence, rather than in terms of capacities operating normally vs. being impaired, there are no common factors of course: the presence of a factor that both makes a difference to, say, the presence of smoke as well as to the absence of it seems very suspect, to say the least. Factors that make a difference to both normal and impaired capacities, say, eyesight and impaired eyesight are unproblematic: an abnormality in the primary visual cortex might explain the contrast yet both normal and impaired eyesight are dependent on retinal function, optic chiasm function, etc. 
haust function, since operation of the exhaust makes a difference to both the excess black smoke and to expulsion of normal amounts of smoke. Also, in order to identify the malfunctioning component - here, say, a rupture in the oil reservoir - you need to understand the normal function of this component as well as the normal functioning of the mechanism in which the component is situated. Malfunction is identified against a backdrop of normal function (cf. Thagard 2003; Moghaddam-Taaheri 2011). Finally, taking common factors such as the hot exhaust into account enables asking and answering more what-if questions than consideration of contrastive factors alone can deliver; e.g., 'what would happen to the black smoke if the temperature of the exhaust were lower?'.

As far as we know, the relevance of this distinction between common and contrastive difference makers has not been appreciated in current mechanistic thinking about explanatory relevance and difference making. ${ }^{7}$

\section{Mechanistic models and difference making}

Mechanistic views on difference making are conceptually related to explanatory virtues. Broadly speaking, two main explanatory virtues are emphasized in the mechanistic explanation literature: 'abstraction' and 'specificity'. Specificity dictates that (ideally) all the entities, activities, and organizational features of mechanisms that are constitutively relevant for the multiple features of a phenomenon to be explained are included in a mechanistic model (Craver 2007). Constitutively relevant factors are ones that make a difference to features of the phenomenon one aims to explain (Craver 2007, 144, 198-211). These multiple features of a phenomenon include "its precipitating conditions, manifestations, inhibiting conditions, modulating conditions, and nonstandard conditions” (Craver 2007, 139).

Constitutively relevant factors are individuated by Craver (2007) in terms of mutual manipulability relationships. On Craver's (2007) account, an entity's activity is considered constitutively relevant to the behaviour of a mechanism as a whole if that entity's activity is a spatiotemporal part of the mechanism, and contributes to the behaviour of the mechanism as a whole. Evidence for constitutive relevance is taken to be procured if one can change the overall behaviour by intervening to change the entity's activity, and if one can change the activity of the entity by intervening to change the overall behaviour. Somewhat more formally, a factor is constitutively relevant if two conditionals are met (Craver 2007, CR1, 155, and CR2, 159): $\mathrm{f}(\varphi 1)$ "

"(CR1) When $\varphi$ is set to the value of $\varphi 1$ in an ideal intervention, then $\psi$ takes on the value $\mathrm{f}(\psi 1)$ "

"(CR2) When $\psi$ is set to the value of $\psi 1$ in an ideal intervention, then $\varphi$ takes on the value

This 'neglect' might have to do with the fact that the role of contrasts, essential to counterfactual accounts of explanation, does not seem vital to most accounts of mechanistic explanation. Mechanistic explanations are often taken to track mechanisms that actually produce phenomena, and typically not construed in counterfactual fashion. Counterfactual reasoning, rather, is often invoked in analyses of mechanism discovery and in explanatory relevance assessments were interventions on putative components are stressed (Craver 2002, 2007). We owe this insight to an anonymous referee. 
These conditionals cover both scenarios in which interventions change the manner in which $\psi$ or $\varphi$ occur, i.e., their value, as well as ones that lead to the occurrence or elimination of $\psi$ or $\phi$ (cf. Craver 2007, 149). In the latter case, $\psi$ or $\phi$ would take on the value ' 1 ' or ' 0 ', respectively. So mutual manipulability relations comprise both constitutive relevance, i.e., difference making, relations with respect to the occurrence of explananda phenomena, as well as relations concerning the precise manner in which explananda phenomena occur (cf. van Eck 2015a). That mutual manipulability comprises two notions of difference making likely relates to the fact that explanatory targets concern 'multiple features' of phenomena, including in addition to manifestations of phenomena, their modulating conditions. ${ }^{8}$

Levy and Bechtel (2013) have recently argued that there are explanatory contexts in which the desideratum of specificity is overkill. They argue that in the work of Craver (2007) and Machamer et al. (2000) structural features of entities too often get assigned explanatory (constitutive) relevance. However, Levy and Bechtel (2013) argue that in the explanatory context of explaining how organization impacts the behaviour of a mechanism, often, skeletal models that suppress reference to structural aspects of components explain better than more elaborate models in which structural features are described. In this context, models that solely describe causal relations between components are best equipped to "explain temporal properties of mechanisms" (p. 241). With respect to such "abstract" models (Levy and Bechtel 2013, 242) they argue that these "track those features of the system that make a difference to the behaviour being explained" (p. 256). ${ }^{9}$ By their lights, the omission of structural details makes salient those causal factors that make a difference to the phenomenon being explained, i.e., (functionally described) components and their causal relations specified in terms of components' causal roles (cf. Levy and Bechtel 2013).

The differences between the 'specificity' and 'abstraction' perspectives can be understood in terms of the different notions of difference making underlying them (cf. van Eck 2015a). As we have seen, in Craver's system two notions of difference making are endorsed: with respect to phenomena occurring and with respect to influencing the manner in which phenomena manifest themselves. Levy and Bechtel, by contrast, can be interpreted as only endorsing difference making in the sense of factors that make a difference to whether or not the explanatory target obtains: they tie their discussion of difference making to Strevens' 'kairetic' account of causal explanation and according to this account, explanatory models should only contain factors that make a difference to whether or not the explanandum obtains, and not factors that merely influence the precise manner in which a phenomenon occurs (Strevens 2004, 2008).

To sum up, both perspectives tie explanatory relevance to 'difference making' yet differ in the ways in which this notion is cashed out. What is relevant for our purposes here

8 Mutual manipulability is not uncontroversial; various extensions and criticisms have been given after Craver's (2007) initial formulation (e.g., Harbecke 2010; Couch 2011; Leuridan 2012; van Eck 2015b; Baumgartner \& Gebharter 2015). Neither of these proposals make a distinction between contrastive and common difference makers though. For reasons spelled out in this paper, explicit recognition of the distinction would make for a relevant extension of these accounts.

9 To be sure, Levy and Bechtel do point out that in other explanatory contexts structural details can be important. 
is that no distinction is made between contrastive and common factors that make a difference. However, what our elaboration of Lipton's (1993) framework tells us is that the distinction is relevant in the context of malfunction explanation, i.e., for diagnostic purposes, mechanism discovery, and (counterfactual) understanding. We detail these points further in terms of a case on malfunction explanations drawn from biology. ${ }^{10}$

\section{Malfunction explanation in (neuro) biology: circadian rhythms and sleeping disorders}

Let us consider the explanation of sleeping disorders as arising from malfunctioning circadian rhythms. Circadian rhythms are endogenous (approximately) 24-hour oscillations governing biological activities such as reproduction, digestion, and maintenance of bodily temperature in a wide range of living organisms. In the case of mammals and birds, they govern sleep as well, where sleep is understood in terms of altered brainwave patterns, reversible unconsciousness, muscle relaxation, and sporadic eye movement. ${ }^{11}$ To use a popular phrase, sleep is governed by a circadian clock. Of course, by itself, the term 'circadian clock' is merely a placeholder for some underlying biochemical mechanism that has yet to be specified, and this mechanism will most likely differ in its precise configuration and implementation across species.

In humans, the biochemical mechanism underlying the sleeping cycle combines external (daylight, food intake, etc.) and internal (e.g. hormonal) factors. The mechanism is called endogenous because it is maintained even in the absence of external cues (Kleitman 1963; Lobban 1961). Nevertheless, in such circumstances the waking-sleeping cycle will start shifting, because left to its own devices, it is slightly longer than 24 hours (Czeisler et al. 1999). Therefore, the mechanism needs the external cues to constantly readjust itself, a process known as entrainment. From these observations, one can infer at least three features of the biochemical mechanism behind our waking-sleeping cycle: photoreceptors conveying information to the internal clock (or pacemaker) for the purposes of entrainment, the clock sub-mechanism itself, and ways for the clock to regulate the systems that ultimately produce the cycle. Here, we already have a basic functional analysis of the mechanism: the overall capacity to maintain a waking-sleeping cycle mirroring the 24-hour cycle of day and night is analysed into three sub-capacities.

${ }^{10}$ Like Craver (2007), we endorse, in the context of our distinction between contrastive and common difference making factors, difference making factors with respect to the occurrence of an explanandum and ones affecting how it occurs. The first notion relates to contrastive factors, the second to common ones. It is a matter of debate whether mutual manipulability is able to track difference makers in the first place. We side with Baumgartner \& Gebharter (2015) that mutual manipulability in itself is not sufficient, but that combined with demonstrating that there are only common causes of a mechanism' overall behaviour and some constituent, and no surgical causes of a mechanism' overall behaviour, this provides sufficient evidence for constitutive difference makers.

11 This is not to say that sleep-like behavior is not observed in other species besides birds and mammals, such as invertebrates and fish, but rather that in these other species it is not (yet or not yet as thoroughly) researched from a physiological perspective - hence the tendency to describe sleep in such species in mostly behavioural terms (non-responsiveness to external stimuli, particular bodily positions, etc.). Here, we are interested in sleep in humans. 
It is also possible to provide some details with regard to the entities performing these activities, so that we get the following explanation for the capacity to maintain a circadian waking-sleeping rhythm. ${ }^{12}$ The first sub-capacity, namely to relay external cues to the internal clock for the purposes of entrainment, is carried out by means of specific cells and pathways. By far the most important external cue (but not the only one) is light (the so-called Zeitgeber). Information about this cue (e.g., light intensity) is passed on from the retina through the retinohypothalamic tract (RHT), which consists of a specialized group of cells located just above the optic chiasm, to the suprachiasmatic nucleus (SCN), a small region of the midline. The SCN functions as the principal structure maintaining the circadian rhythm (the internal clock). Entrainment of the SCN in accordance with the external cues is managed by a series of complex feedback loops, involving (among other things), the hormone melatonin. This hormone is produced by the pineal gland at night in a rhythm controlled by the SCN, and then feeds back into the SCN (which contains melatonin receptors), thus establishing a feedback loop by means of which entrainment can take place. Next, the SCN signals the hypothalamus by a rhythmic neuronal firing, the rate of which is high during the day, and low during the night. In turn, the hypothalamus sends circadian information to several regions such as the anterior pituitary, the hypothalamus, and the brainstem reticular formation, which (among other things) regulate the waking-sleeping cycles.

Thus, our ability to maintain a circadian waking-sleeping cycle is realized by a complex biochemical mechanism. Although this model is far more specific than the previous functional one, which only analysed the target capacity into three sub-capacities, it is still to a degree abstract. It glosses over many more fine-grained mechanisms. For example, just how the hypothalamus converts the signals coming in from the SCN into circadian information is governed by yet another complex mechanism. Including all the information we now know about the circadian rhythms governing the waking-sleeping cycle into our model would result in a dense hierarchical characterization of mechanisms nested within mechanisms.

In the case at hand, sleeping disorders provide examples of malfunctioning circadian systems-mechanisms. As one would expect, the mechanism described above can break down in a number of places; and as we have already analysed the overall capacity to maintain a circadian waking-sleeping cycle into three sub-capacities (entrainment, the clock or pacemaker, and the pacemaker regulating the cycles), it is possible to classify sleeping disorders into a corresponding number of types. ${ }^{13}$ For example, some sleeping disorders arise from a malfunction in the sub-capacity of entrainment (e.g., blindness/24-hour sleep-wake syndrome, work-shift syndrome, delayed phase sleep syndrome, advanced phase sleep syndrome etc.).

In turn, entrainment sleeping disorders can be caused by the external cues being blocked or distorted, or by the RHT failing to pass on information, or by some of the hormonal feedback loops being impaired. In explaining a particular entrainment disorder, it is necessary of course to describe such contrastive difference makers that underlie the entrainment sleeping disorder, say, the manner in which external cues in the pathways in between the retina and RHT get distorted, or which structural abnormalities in the RHT prevent

12 The following model is adapted from Moore (1997).

13 We restrict our analysis to those mechanisms that are decomposable into subsystems-mechanisms and which can have localizable malfunctions of those subsystems-mechanisms. This differs from Moghaddam-Taaheri's (2011) analysis of a case of cystic fibrosis in which a gene mutation affects all the downstream components. We thank an anonymous referee for suggesting this clarification. 
information to be passed on to the SCN. But here is the point: consideration of the other sub-capacities that underlie both normal function and the malfunction that one aims to explain is relevant as well. Say, in the case of a malfunctioning pathway in between the retina and the RHT, the retina and the RHT themselves are also included in the explanation, together with the fact that the latter does not receive the information it normally does. Similarly, when a structural deformation prevents the RHT to pass on information to the SCN, not only the specifics of the structural abnormality are important but also that the SCN does not receive information. The SCN is clearly a difference maker of the second category, since it is not only a relevant entity to explain entrainment sleeping disorders, for normal sleep function also depends on it.

Distinguishing contrastive difference makers from factors common to both malfunction and normal functioning is relevant for repair (or therapeutic intervention) purposes, since you only want to tinker with factors of the first category. You only want to intervene on, say, the deformation that prevents the RHT to transfer information to the SCN, not on the SCN itself. Furthermore, therapeutic interventions can only be made, and similarly, explanations provided, when a malfunctioning mechanism is individuated correctly, that is, when both contrastive and common factors have been identified and the malfunction (contrastive factor) has been localized in a mechanism (cf. Thagard 2003). You need to know how, say, retina and RHT are connected in order to identify normal function(s) and malfunction(s) of the pathway connecting them. Finally, taking both contrastive and common factors into account offers deeper understanding, in the sense of what-if-questions, of malfunctioning systems-mechanisms than consideration of contrastive factors alone (cf. Woodward 2003). Compare 'what would happen if the structural abnormality/deformation of the RHT was changed in such or so fashion?' versus 'what would happen if this particular change was effected?' and 'what effect would this change have on information transfer to the SCN?'. The latter set of questions and answers probes deeper into a malfunctioning system-mechanism, than understanding solely procured in terms of an answer to the former question.

Summing up, when considering mechanistic explanations for malfunctions, extending the notion of difference making to include common and contrastive difference makers has significant advantages for repair/therapeutic intervention purposes, mechanism discovery, and (counterfactual) understanding. ${ }^{14}$

\section{REFERENCES}

Baumgartner, M. \& A. Gebharter. 2015. Constitutive Relevance, Mutual Manipulability, and Fat-handedness. Forthcoming in The British Journal for the Philosophy of Science.

Couch, M. 2011. Mechanisms and Constitutive Relevance. Synthese 183: 375-88.

Craver, C.F. 2001. Role functions, Mechanisms, and Hierarchy. Philosophy of Science 68, 53-74.

Craver, C.F. 2002. Interlevel Experiments and Multilevel Mechanisms in the Neuroscience of Memory. Philosophy of Science 69, S83-S97.

Craver, C.F. 2006. When Mechanistic Models Explain. Synthese 153: 355-76.

${ }^{14}$ As will be evident, common factors are 'higher order' factors, not 'primary' ones that make a difference to whether or not the explanatory target obtains (cf. notes 3 and 6 ). 
Craver, C.F. 2007. Explaining the Brain: Mechanisms and the Mosaic Unity of Neuroscience. New York: Oxford University Press.

Craver, C.F. 2013. Functions and Mechanisms: A Perspectivalist View. In Functions: Selection and Mechanisms, ed. P. Huneman, 133-58. Dordrecht: Springer.

Czeisler, C.A. et al. 1999. Stability, Precision, and near-24-hour Period of Human Circadian Pacemaker. Science 5423 (284): 2177-2181.

Glennan, S. 2005. Modeling Mechanisms. Studies in History and Philosophy of the Biological and Biomedical Sciences 36 (2): 375-88.

Harbecke, J. 2010. Mechanistic Constitution in Neurobiological Explanations. International Studies in the Philosophy of Science 24: 267-85.

Illari, P. \& J. Williamson. 2010. Function and Organization: Comparing the Mechanisms of Protein Synthesis and Natural Selection. Studies in History and Philosophy of Biological and Biomedical Sciences 41: 279-91.

Kleitman, N. 1963. Sleep and wakefulness. Chicago: University of Chicago Press.

Leuridan, B. 2012. Three Problems for the Mutual Manipulability Account of Constitutive Relevance in Mechanisms. The British Journal for the Philosophy of Science 63 (2): 399-427.

Levy, A. \& W. Bechtel. 2013. Abstraction and the Organization of Mechanisms. Philosophy of Science 80: 241-61.

Lipton, P. 1993. Making a Difference. Philosophica 51:39-54.

Lobban, M.C. 1961. The Entrainment of Circadian Rhythms in Man. Cold Spring Harbor Symposia on Quantitative Biology 25: 325-32.

Machamer, P.K.; Darden, L. \& C.F. Craver. 2000. Thinking About Mechanisms. Philosophy of Science 57: 1-25.

Moghaddam-Taaheri, S. 2011. Understanding Pathology in the Context of Physiological Mechanisms: The Practicality of a Broken-Normal View. Biology and Philosophy 26: 603-611.

Moore, R.Y. 1997. Circadian Rhythms: Basic Neurobiology and Clinical Applications. Annual Review of Medicine 48: 253-66.

Strevens, M. 2004. The Causal and Unification Approaches to Explanation Unified-Causally. Noûs 38 (1): 154-76.

Strevens, M. 2008. Depth: an Account of Scientific Explanation. Cambridge, MA: Harvard University Press.

Thagard, P. 2003. Pathways to Biomedical Discovery. Philosophy of science 70: 235-54.

van Eck, D. 2015a. Mechanistic Explanation in Engineering Science. European Journal for Philosophy of Science (5): 349-375.

van Eck, D. 2015b. Reconciling Ontic and Epistemic Constraints on Mechanistic Explanation, Epistemically. Axiomathes 25 (1): 5-22.

Waters, C.K. 2007. Causes that Make a Difference. The Journal of Philosophy 104 (11): 551-79.

Weisberg, M. 2007. Three Kinds of Idealization. The Journal of Philosophy, 104 (12): 639-59.

Woodward, J. 2003. Making Things Happen. Oxford: Oxford University Press.

Dingmar van Eck is postdoctoral researcher in philosophy of science at the Centre for Logic and Philosophy of Science, Ghent University. Most of his current research is on issues related to scientific explanation in the life sciences and engineering sciences.

Address: Centre for Logic and Philosophy of Science, Ghent University, Blandijnberg 2,B-9000 Gent, Belgium. Email: Dingmar.vanEck@Ugent.be

Raoul Gervais is postdoctoral fellow of the Research Foundation Flanders (FWO) at the University of Antwerp's Centre for Philosophical Psychology, and assistant professor at the Tilburg Center for Logic, Ethics, and Philosophy of Science. Currently, his research focuses on causality and explanation in the mind/ brain sciences.

Address: The University of Antwerp's Centre for Philosophical Psychology (University of Antwerp), Grote Kauwenberg 18, 2000 Antwerp (Belgium). Email: Raoul.Gervais@Uantwerpen.be 